\title{
Semileptonic $\Lambda_{b} \rightarrow \Lambda_{c} \ell \bar{\nu}_{\ell}$ transition in full QCD
}

\author{
K. Azizi ${ }^{1,2}$ and J. Y. Süngü ${ }^{3}$ \\ ${ }^{1}$ School of Physics, Institute for Research in Fundamental Sciences (IPM), \\ P.O. Box 19395-5531, Tehran, Iran \\ ${ }^{2}$ Physics Department, Doğuş University, Acıbadem-Kadıköy, 34722 Istanbul, Turkey \\ ${ }^{3}$ Department of Physics, Kocaeli University, 41380 Izmit, Turkey
}

(Received 6 March 2018; published 9 April 2018)

\begin{abstract}
The tree-level $b \rightarrow c \ell \bar{\nu}_{\ell}$ based hadronic transitions have been the focus of much attention since recording significant deviations of the experimental data, on the ratios of the branching fractions in $\tau$ and $e-\mu$ channels of the semileptonic $B \rightarrow D$ transition, from the SM predictions by the BABAR Collaboration in 2012. It can be of great importance to look whether similar discrepancies take place in the semileptonic baryonic $\Lambda_{b} \rightarrow \Lambda_{c} \ell \bar{\nu}_{\ell}$ decay channel or not. In this accordance we estimate the decay width as well as the ratios of the branching fractions in $\tau$ and $e-\mu$ channels of this baryonic transition by calculating the form factors, entering the amplitude of this transition as the main inputs, in the framework of QCD sum rules in full theory. We compare the obtained results with the predictions of other theoretical studies. Our results may be compared with the corresponding future experimental data to look for possible deviations of data from the SM predictions.
\end{abstract}

DOI: 10.1103/PhysRevD.97.074007

\section{INTRODUCTION}

One of the main goals of the LHC, after the discovery of Higgs, is to search for the new physics (NP) effects. This is done via two ways: direct search at colliders and indirect search for the NP effects in hadronic decay channels. Recently, there have been recorded significant deviations from the SM predictions: the BABAR measurements [1] on the ratios of the branching fractions of the semileptonic $B \rightarrow D$ decay in $\tau$ channel to those of the $e$ or $\mu$ had shown to deviate at the global level of $3.4 \sigma$ from the SM predictions [2,3]. One of the most important results newly obtained at LHC is the sign of the lepton flavor universality violation (LFUV) in the semileptonic $B$ decays. The LHC data [4] on

$$
\begin{aligned}
R_{k} & =\frac{\operatorname{Br}\left(B^{+} \rightarrow K^{+} \mu \mu\right)}{\operatorname{Br}\left(B^{+} \rightarrow K^{+} e e\right)} \\
& =0.745_{-0.074}^{+0.090}(\text { stat }) \pm 0.036(\text { syst })
\end{aligned}
$$

lies $2.6 \sigma$ below the SM prediction in the window $q^{2} \in[1,6] \mathrm{GeV}^{2}$. Similar indications have been newly reported in semileptonic decay, $B \rightarrow K$ [5]. Hence, the semileptonic $B$ decays seem to be good probe to search for

Published by the American Physical Society under the terms of the Creative Commons Attribution 4.0 International license. Further distribution of this work must maintain attribution to the author(s) and the published article's title, journal citation, and DOI. Funded by SCOAP . the new physics beyond the SM. In principle, similar behaviors and deviations from the SM predictions can occur in other b-hadron decays. In [6], it was shown that some experimental data on the differential branching ratio as well as lepton forward-backward asymmetry in $\Lambda_{b} \rightarrow \Lambda \mu^{+} \mu^{-}$channel cannot be described by the SM predictions provided by the lattice QCD and QCD sum rules. Although there previously were predictions of different models in heavy quark effective theory limit, the form factors of $\Lambda_{b} \rightarrow \Lambda \ell^{+} \ell^{-}$were first calculated in 2010 in full theory including all twelve form factors in Ref. [7]. The obtained results on the order of branching fractions at different lepton channels had shown that these channels were accessible at hadron colliders. One year later, the CDF Collaboration observed this baryonic flavor-changing neutral current decay in $\mu$ channel [8]. In 2015 the LHCb Collaboration measured the differential branching ratio and made angular analysis of the same decay mode [9]. In 2016, the lattice predictions became available, where the form factors, differential branching fraction, and angular observables with relativistic $\mathrm{b}$ quarks associated to this channel were calculated [10]. Considering the new experimental developments on the spectroscopy and decays properties of heavy hadrons, it seems that the b-baryon decays, especially the $\Lambda_{b}$ baryon decay modes become important not only for exact determinations of different SM parameters but as essential sources of the physics BSM: very recently the $\mathrm{LHCb}$ Collaboration has found evidence for $C P$ violation in $\Lambda_{b}$ to $p \pi^{-} \pi^{+} \pi^{-}$decays with a statistical significance corresponding to 3.3 standard deviations 
including systematic uncertainties. This represents the first evidence for $C P$ violation in the baryon sector [11].

Many parameters related to different decay channels of the $\Lambda_{b}$ state have been previously studied using different approaches such as relativistic quark model, soft-collinear effective theory, heavy quark effective theory, covariant quark model, zero recoil sum rule, lattice QCD and QCD sum rules (see for instance Refs. [7,12-26] and references therein). The tree-level $b \rightarrow c \ell \bar{\nu}_{\ell}$ based semileptonic $\Lambda_{b} \rightarrow$ $\Lambda_{c} \ell \bar{\nu}_{\ell}$ transition is one of the prominent decay channels of the $\Lambda_{b}$ baryon. This channel has been investigated using different quark models and lattice QCD [16-24]. We analyze this decay in $e, \mu$, and $\tau$ channels. In particular we calculate all six form factors entering the matric elements of the effective Hamiltonian sandwiched between the initial and final baryonic states in full QCD without making the heavy quark effective theory approximation. We calculate the decay width and branching ratios in all lepton channels and compare the results with the predictions of other theoretical approaches as well as existing experimental data. We compute the ratio of the branching fractions in $\tau$ channel to those of the $e$ or $\mu$ associated to this transition, as well.

This paper is organized as follows. In Sec. II, we calculate the six form factors defining the $\Lambda_{b} \rightarrow \Lambda_{c} \ell \bar{\nu}_{\ell}$ transition using the technique of QCD sum rules [27]. In Sec. III we numerically analyze the form factors and find their $q^{2}$-dependent fit functions. Section IV is devoted to calculations of different physical observables related to the decays under consideration and comparison of the results obtained with the predictions of other theoretical studies as well as existing experimental data. Section $\mathrm{V}$ is reserved for our concluding remarks and, finally, we move some analytic expressions for the spectral densities used in the calculations to the Appendix.

\section{TRANSITION FORM FACTORS}

The $\Lambda_{b} \rightarrow \Lambda_{c} \ell \bar{\nu}_{\ell}$ decay channel proceeds via $b \rightarrow c \ell \bar{\nu}_{\ell}$ at quark level. The low-energy effective Hamiltonian describing this transition can be written as

$$
\mathcal{H}_{\mathrm{eff}}=\frac{G_{F}}{\sqrt{2}} V_{c b} \bar{c} \gamma_{\mu}\left(1-\gamma_{5}\right) b \bar{l} \gamma^{\mu}\left(1-\gamma_{5}\right) \nu,
$$

where $G_{F}$ is the Fermi coupling constant and $V_{c b}$ is one of the elements of the CKM matrix. The amplitude of this channel is found by sandwiching this effective Hamiltonian between the initial and final baryonic state,

$$
M=\left\langle\Lambda_{c}\left|\mathcal{H}_{\mathrm{eff}}\right| \Lambda_{b}\right\rangle
$$

where the pointlike particles immediately go out of the matrix element and remaining parts are parametrized in terms of six form factors $F_{1}\left(q^{2}\right), F_{2}\left(q^{2}\right), F_{3}\left(q^{2}\right)$, and $G_{1}\left(q^{2}\right), G_{2}\left(q^{2}\right), G_{3}\left(q^{2}\right)$ in full QCD:

$$
\begin{aligned}
& \left\langle\Lambda_{c}\left(p^{\prime}, s^{\prime}\right)\left|V^{\mu}\right| \Lambda_{b}(p, s)\right\rangle \\
& =\bar{u}_{\Lambda_{c}}\left(p^{\prime}, s^{\prime}\right)\left[F_{1}\left(q^{2}\right) \gamma^{\mu}+F_{2}\left(q^{2}\right) \frac{p^{\mu}}{m_{\Lambda_{b}}}+F_{3}\left(q^{2}\right) \frac{p^{\prime \mu}}{m_{\Lambda_{c}}}\right] \\
& \quad \times u_{\Lambda_{b}}(p, s), \\
& \left\langle\Lambda_{c}\left(p^{\prime}, s^{\prime}\right)\left|A^{\mu}\right| \Lambda_{b}(p, s)\right\rangle \\
& =\bar{u}_{\Lambda_{c}}\left(p^{\prime}, s^{\prime}\right)\left[G_{1}\left(q^{2}\right) \gamma^{\mu}+G_{2}\left(q^{2}\right) \frac{p^{\mu}}{m_{\Lambda_{b}}}+G_{3}\left(q^{2}\right) \frac{p^{\prime \mu}}{m_{\Lambda_{c}}}\right] \\
& \quad \times \gamma_{5} u_{\Lambda_{b}}(p, s) .
\end{aligned}
$$

In above equation, $V^{\mu}=\bar{c} \gamma_{\mu} b$ and $A^{\mu}=\bar{c} \gamma_{\mu} \gamma_{5} b$ are the vector and axial vector parts of the transition current, $q=p-p^{\prime}$ is the momentum transferred to the leptons; and $u_{\Lambda_{c}}(p, s)$ and $u_{\Lambda_{c}}\left(p^{\prime}, s^{\prime}\right)$ are Dirac spinors of the initial and final baryonic states.

The main goal in the following is to calculate the six transition form factors in full QCD using the technique of the three-point sum rule as one of the powerful and applicable nonperturbative tools to hadron physics. As usual prescriptions, the starting point is to consider an appropriate correlation function of interpolating and transition currents in a time ordered manner. The sum rules for transition form factors are found by equating the phenomenological or physical representation of this three point function to the theoretical or QCD side of the same function which is obtained using the operator product expansion (OPE). The three-point correlation function for our aim is

$$
\begin{aligned}
\Pi_{\mu}\left(p, p^{\prime}, q\right)= & i^{2} \int d^{4} x e^{-i p \cdot x} \int d^{4} y e^{i p^{\prime} \cdot y} \\
& \times\left\langle 0|\mathcal{T}| \mathcal{J}^{\Lambda_{c}}(y) \mathcal{J}_{\mu}^{t r, V(A)}(0) \mathcal{J}^{\dagger \Lambda_{b}}(x) \mid 0\right\rangle,
\end{aligned}
$$

where $\mathcal{T}$ is the time-ordering operator, $\mathcal{J}_{\mu}^{\mathrm{tr}, V(A)}=$ $\bar{c} \gamma_{\mu} b\left(\bar{c} \gamma_{\mu} \gamma_{5} b\right)$ is the vector (axial-vector) part of the transition current and $\mathcal{J}^{\Lambda_{Q}}(x)$ with $Q$ being $b$ or $c$ quark is the interpolating current for the $\Lambda_{b}$ or $\Lambda_{c}$ baryon. It is given in its more general form as:

$$
\begin{aligned}
\mathcal{J}^{\Lambda_{Q}}(x)= & \frac{1}{\sqrt{6}} \epsilon_{a b c}\left\{2\left[\left(q_{1}^{a T}(x) C q_{2}^{b}(x)\right) \gamma_{5} Q^{c}(x)+\beta\left(q_{1}^{a T}(x) C \gamma_{5} q_{2}^{b}(x)\right) Q^{c}(x)\right]+\left(q_{1}^{a T}(x) C Q^{b}(x)\right) \gamma_{5} q_{2}^{c}(x)\right. \\
& \left.+\beta\left(q_{1}^{a T}(x) C \gamma_{5} Q^{b}(x)\right) q_{2}^{c}(x)+\left(Q^{a T}(x) C q_{2}^{b}(x)\right) \gamma_{5} q_{1}^{c}(x)+\beta\left(Q^{a T}(x) C \gamma_{5} q_{2}^{b}(x)\right) q_{1}^{c}(x)\right\}
\end{aligned}
$$


where $a, b$ and $c$ are color indices, $C$ is the charge conjugation operator, $q_{1}$ and $q_{2}$ are $u$ and $d$ quark fields, respectively. The $\beta$ is a general mixing parameter with $\beta=-1$ being corresponding to Ioffe current. The physical or phenomenological side is found by inserting complete sets of the initial and final baryonic states with the same quantum numbers as the interpolating currents into the correlation function. By performing integrals over four- $x$ and $-y$ we end up with

$$
\Pi_{\mu}^{\text {Phys }}\left(p, p^{\prime}, q\right)=\frac{\left\langle 0\left|\mathcal{J}^{\Lambda_{c}}(0)\right| \Lambda_{c}\left(p^{\prime}\right)\right\rangle\left\langle\Lambda_{c}\left(p^{\prime}\right)\left|\mathcal{J}_{\mu}^{\mathrm{tr}, V(A)}(0)\right| \Lambda_{b}(p)\right\rangle\left\langle\Lambda_{b}(p)\left|\mathcal{J}^{\dagger \Lambda_{b}}(0)\right| 0\right\rangle}{\left(p^{\prime 2}-m_{\Lambda_{c}}^{2}\right)\left(p^{2}-m_{\Lambda_{b}}^{2}\right)}+\cdots
$$

where $\cdots$ stands for the contributions of the higher states and continuum. Besides the transition matrix elements we need to define the following matrix elements in terms of the residues of the initial and final states:

$$
\begin{aligned}
\left\langle 0\left|\mathcal{J}^{\Lambda_{c}}(0)\right| \Lambda_{c}\left(p^{\prime}\right)\right\rangle & =\lambda_{\Lambda_{c}} u_{\Lambda_{c}}\left(p^{\prime}, s^{\prime}\right), \\
\left\langle\Lambda_{b}(p)\left|\overline{\mathcal{J}}^{\Lambda_{b}}(0)\right| 0\right\rangle & =\lambda_{\Lambda_{b}}^{+} \bar{u}_{\Lambda_{b}}(p, s) .
\end{aligned}
$$

The final step is to put all the matrix elements defined above into Eq. (7) and use the summation over Dirac spinors

$$
\begin{aligned}
u_{\Lambda_{c}}\left(p^{\prime}, s^{\prime}\right) \bar{u}_{\Lambda_{c}}\left(p^{\prime}, s^{\prime}\right) & =\not p^{\prime}+m_{\Lambda_{c}}, \\
u_{\Lambda_{b}}(p, s) \bar{u}_{\Lambda_{b}}(p, s) & =\not p+m_{\Lambda_{b}} .
\end{aligned}
$$

As a result we find the following representation for the final form of the physical side in terms of the structures used in the calculations in Borel transformed form that has been applied to suppress the contributions of the higher states and continuum:

$$
\begin{aligned}
\hat{\mathbf{B}} \Pi_{\mu}^{\mathrm{Phys}}\left(p, p^{\prime}, q\right) & \\
= & {\left[m_{\Lambda_{b}} F_{1} \not p^{\prime} \gamma_{\mu}+\frac{1}{m_{\Lambda_{b}}} F_{2} p_{\mu} \not \not^{\prime} \not p+\frac{1}{m_{\Lambda_{c}}} F_{3} p_{\mu}^{\prime} \not \not^{\prime} \not p\right.} \\
& +m_{\Lambda_{b}} m_{\Lambda_{c}} G_{1} \gamma_{\mu} \gamma_{5}-\frac{1}{m_{\Lambda_{b}}} G_{2} p_{\mu} \not p^{\prime} \not \gamma_{5} \\
& \left.-\frac{1}{m_{\Lambda_{b}}} G_{3} p_{\mu}^{\prime} \not p^{\prime} \not \gamma_{5}+\cdots\right] \lambda_{\Lambda_{b}} \lambda_{\Lambda_{c}} e^{-\frac{m_{\Lambda_{b}}^{2}}{M^{2}}} e^{-\frac{m_{\Lambda_{c}}^{2}}{M^{2}}},
\end{aligned}
$$

where $M^{2}$ and $M^{\prime 2}$ are Borel parameters that should be fixed later and we kept only the structures that will be used in further analyses.

To find the correlation function in terms of quark-gluon degrees of freedom on QCD side, i.e., by utilizing the light and heavy propagators, we use the interpolating current given by Eq. (6) in Eq. (5), and contract the related quark fields. After some manipulations including the contraction of the quark fields, we find the QCD side in terms of the heavy and light quarks' propagators in coordinate space. Thus, for the light quark we use

$$
\begin{aligned}
S_{q}^{a b}(x)= & i \delta_{a b} \frac{\not x}{2 \pi^{2} x^{4}}-\delta_{a b} \frac{m_{q}}{4 \pi^{2} x^{2}}-\delta_{a b} \frac{\langle\bar{q} q\rangle}{12}+i \delta_{a b} \frac{\not m_{q}\langle\bar{q} q\rangle}{48} \\
& -\delta_{a b} \frac{x^{2}}{192}\langle\bar{q} g \sigma G q\rangle+i \delta_{a b} \frac{x^{2} \not m_{q}}{1152}\langle\bar{q} g \sigma G q\rangle \\
& -i \frac{g G_{a b}^{\alpha \beta}}{32 \pi^{2} x^{2}}\left[\not x \sigma_{\alpha \beta}+\sigma_{\alpha \beta} \not x\right]-i \delta_{a b} \frac{x^{2} \not g^{2}\langle\bar{q} q\rangle^{2}}{7776} \\
& -\delta_{a b} \frac{x^{4}\langle\bar{q} q\rangle\left\langle g^{2} G^{2}\right\rangle}{27648}+\cdots,
\end{aligned}
$$

and the heavy quark propagator is given as [28];

$$
\begin{aligned}
S_{Q}^{a b}(x)= & i \int \frac{d^{4} k}{(2 \pi)^{4}} e^{-i k x}\left\{\frac{\delta_{a b}\left(\not k+m_{Q}\right)}{k^{2}-m_{Q}^{2}}\right. \\
& -\frac{g G_{a b}^{\alpha \beta}}{4} \frac{\sigma_{\alpha \beta}\left(\not k+m_{Q}\right)+\left(\not k+m_{Q}\right) \sigma_{\alpha \beta}}{\left(k^{2}-m_{Q}^{2}\right)^{2}} \\
& \left.+\frac{g^{2} G^{2}}{12} \delta_{a b} m_{Q} \frac{k^{2}+m_{Q} \not k}{\left(k^{2}-m_{Q}^{2}\right)^{4}}+\cdots\right\},
\end{aligned}
$$

where we used the following notations

$$
G_{a b}^{\alpha \beta}=G_{A}^{\alpha \beta} t_{a b}^{A}, \quad G^{2}=G_{\alpha \beta}^{A} G_{\alpha \beta}^{A},
$$

with $a, b=1,2,3$ being the color and $A, B, C=1,2 \ldots 8$ being the flavor indices. In Eq. (13) $t^{A}=\lambda^{A} / 2, \lambda^{A}$ are the Gell-Mann matrices and the gluon field strength tensor $G_{\alpha \beta}^{A} \equiv G_{\alpha \beta}^{A}(0)$ is fixed at $x=0$.

By replacing the heavy and light quark propagators we apply the following Fourier transformation:

$$
\begin{aligned}
\frac{1}{\left[(y-x)^{2}\right]^{n}}= & \int \frac{d^{D} t}{(2 \pi)^{D}} e^{-i t \cdot(y-x)} i(-1)^{n+1} 2^{D-2 n} \pi^{D / 2} \\
& \times \frac{\Gamma(D / 2-n)}{\Gamma(n)}\left(-\frac{1}{t^{2}}\right)^{D / 2-n} .
\end{aligned}
$$

Then, the four-dimensional $x$ and $y$ integrals are performed in the sequel of the replacements $x_{\mu} \rightarrow i \frac{\partial}{\partial p_{\mu}}$ and $y_{\mu} \rightarrow-i \frac{\partial}{\partial p_{\mu}^{\prime}}$. This procedure brings two four-dimensional Dirac delta functions which are used to perform the fourintegrals over $k$ and $k^{\prime}$ coming from the heavy $b$ and $c$ quarks propagators. Then the Feynman parametrization and 


$$
\int d^{4} t \frac{\left(t^{2}\right)^{\beta}}{\left(t^{2}+L\right)^{\alpha}}=\frac{i \pi^{2}(-1)^{\beta-\alpha} \Gamma(\beta+2) \Gamma(\alpha-\beta-2)}{\Gamma(2) \Gamma(\alpha)[-L]^{\alpha-\beta-2}},
$$

are used to carry out the remaining four-integral over $t$. The function $\Pi_{\mu}^{\mathrm{QCD}}\left(p, p^{\prime}, q\right)$ includes twenty-four different structures that not all of them are written here:

$$
\begin{aligned}
\Pi_{\mu}^{\mathrm{QCD}}\left(p, p^{\prime}, q\right)= & \Pi_{p^{\prime} \gamma_{\mu}}^{\mathrm{QCD}}\left(p^{2}, p^{2}, q^{2}\right) \not p^{\prime} \gamma_{\mu} \\
& +\Pi_{p_{\mu} p^{\prime} p}^{\mathrm{QCD}}\left(p^{2}, p^{\prime 2}, q^{2}\right) p_{\mu} \not \not^{\prime} \not p+\cdots,
\end{aligned}
$$

where, the invariant functions $\Pi_{i}^{\mathrm{QCD}}\left(p^{2}, p^{\prime 2}, q^{2}\right)$, with $i$ representing different structures, are represented in terms of a double dispersion integral as

$$
\begin{aligned}
\Pi_{i}^{\mathrm{QCD}}\left(p^{2}, p^{\prime 2}, q^{2}\right)= & \int_{s_{\min }}^{\infty} d s \int_{s_{\min }^{\prime}}^{\infty} d s^{\prime} \frac{\rho_{i}^{\mathrm{QCD}}\left(s, s^{\prime}, q^{2}\right)}{\left(s-p^{2}\right)\left(s^{\prime}-p^{\prime 2}\right)} \\
& + \text { subtracted terms, }
\end{aligned}
$$

where $s_{\min }=\left(m_{u}+m_{d}+m_{b}\right)^{2}, s_{\min }^{\prime}=\left(m_{u}+m_{d}+m_{c}\right)^{2}$ and $\rho_{i}^{\mathrm{QCD}}\left(s, s^{\prime}, q^{2}\right)$ are the spectral densities corresponding to different structures. These spectral densities that are obtained by taking the imaginary parts of the $\Pi_{i}^{\mathrm{QCD}}\left(p^{2}, p^{2}, q^{2}\right)$ functions according to the standard prescriptions of the method used, include two different parts and can be classified as

$$
\rho_{i}^{\mathrm{QCD}}\left(s, s^{\prime}, q^{2}\right)=\rho_{i}^{\text {Pert. }}\left(s, s^{\prime}, q^{2}\right)+\sum_{n=3}^{5} \rho_{i}^{n}\left(s, s^{\prime}, q^{2}\right),
$$

where by $\rho_{i}^{n}\left(s, s^{\prime}, q^{2}\right)$ we denote the nonperturbative contributions to $\rho_{i}^{\mathrm{QCD}}\left(s, s^{\prime}, q^{2}\right): n=3,4$ and 5 stand for the quark, gluon and mixed condensates, respectively. Due to the lengthy expressions of the spectral densities, we present only the explicit forms of the spectral densities $\rho_{\not^{\prime} \gamma_{\mu}}^{\text {Pert }}\left(s, s^{\prime}, q^{2}\right)$ and $\rho_{\not p^{\prime} \gamma_{\mu}}^{n}\left(s, s^{\prime}, q^{2}\right)$ corresponding to the Dirac structure $\not p^{\prime} \gamma_{\mu}$ in the Appendix.

After applying the double Borel transformation on the variables $p^{2}$ and $p^{\prime 2}$ in QCD side and subtracting the contribution of the higher resonances and continuum states supported by the quark-hadron duality assumption and matching the coefficients of different structures from the physical and QCD sides of the correlation function, we find the required sum rules for the form factors that will be used in numerical calculations.

\section{NUMERICAL RESULTS FOR FORM FACTORS}

In this section, we shall give our numerical results for the form factors and find their fit functions in terms of $q^{2}$. In our calculations, we set $m_{u}$ and $m_{d}$ equal to zero. Other input parameters used in our evaluation are collected in
TABLE I. Input parameters used in calculations.

\begin{tabular}{lc}
\hline \hline Parameters & Values \\
\hline$m_{c}$ & $(1.28 \pm 0.03) \mathrm{GeV}[29]$ \\
$m_{b}$ & $\left(4.18_{2.29}^{0.04}\right) \mathrm{GeV}[29]$ \\
$m_{e}$ & $0.00051 \mathrm{GeV}[29]$ \\
$m_{\mu}$ & $0.1056 \mathrm{GeV}[29]$ \\
$m_{\tau}$ & $1.776 \mathrm{GeV}[29]$ \\
$m_{\Lambda_{b}}$ & $(5619.51 \pm 0.23) \mathrm{MeV}[29]$ \\
$m_{\Lambda_{c}}$ & $(2286.46 \pm 0.14) \mathrm{GeV}[29]$ \\
$G_{F}$ & $1.17 \times 10^{-5} \mathrm{GeV}^{-2}[29]$ \\
$V_{c b}$ & $(39 \pm 1.1) \times 10^{-3}[29]$ \\
$m_{0}^{2}$ & $(0.8 \pm 0.2) \mathrm{GeV}^{2}[30,31]$ \\
$\tau_{\Lambda_{b}}$ & $1.47 \times 10^{-12}[29]$ \\
$\langle u \bar{u}\rangle=\langle d \bar{d}\rangle$ & $(0.24 \pm 0.01)^{3} \mathrm{GeV}^{3}[32]$ \\
$\left\langle 0\left|\frac{1}{\pi} \alpha_{s} G^{2}\right| 0\right\rangle$ & $(0.012 \pm 0.004) \mathrm{GeV}^{4}[32]$ \\
\hline \hline
\end{tabular}

Table I. The sum rules for form factors contain extra four auxiliary parameters: the Borel parameters $M^{2}$ and $M^{\prime 2}$ as well as the continuum thresholds $s_{0}$ and $s_{0}^{\prime}$. According to the standard prescriptions of the method, the results of form factors should be practically independent of these parameters. Hence their working regions are settled such that the results of form factors depend possibly weakly on these parameters.

The continuum thresholds $s_{0}$ and $s_{0}^{\prime}$ are not entirely arbitrary parameters and they are in correlation with the energy of the first excited states in the initial and final channels. We choose then the intervals

$$
\left(m_{\Lambda_{b}}+0.1\right)^{2} \mathrm{GeV}^{2} \leq s_{0} \leq\left(m_{\Lambda_{b}}+0.5\right)^{2} \mathrm{GeV}^{2},
$$

and

$$
\left(m_{\Lambda_{c}}+0.1\right)^{2} \mathrm{GeV}^{2} \leq s_{0}^{\prime} \leq\left(m_{\Lambda_{c}}+0.5\right)^{2} \mathrm{GeV}^{2} .
$$

The working regions for the Borel mass parameters are determined such that the results show good stability with respect to the variations of these auxiliary parameters. The are a lot of ways to fix these mathematical quantities (see [33] for one of these ways). To find the working regions of these parameters we apply the requirements that not only the higher state and continuum contributions are suppressed but also the contributions of the higher order operators are small, i.e., the sum rules are convergent. Thus, the upper bound of the Borel parameters are found demanding that the ground state contributions in the initial and final channels exceed the contributions of the higher states and continuum, i.e., we impose the condition

$$
\frac{\int_{s_{\min }}^{s_{0}} d s \int_{s_{\min }^{\prime}}^{s_{0}^{\prime}} d s^{\prime} e^{-s / M^{2}} e^{-s^{\prime} / M^{\prime 2}} \rho_{i}\left(s, s^{\prime}, q^{2}\right)}{\int_{s_{\min }}^{\infty} d s \int_{s_{\min }^{\prime}}^{\infty} d s^{\prime} e^{-s / M^{2}} e^{-s^{\prime} / M^{\prime 2}} \rho_{i}\left(s, s^{\prime}, q^{2}\right)}>\frac{1}{2} .
$$


TABLE II. Parameters of the fit function for different form factors corresponding to $\Lambda_{b} \rightarrow \Lambda_{c}$ transition.

\begin{tabular}{|c|c|c|c|c|c|c|}
\hline & $F_{1}\left(q^{2}\right)$ & $F_{2}\left(q^{2}\right)$ & $F_{3}\left(q^{2}\right)$ & $G_{1}\left(q^{2}\right)$ & $G_{2}\left(q^{2}\right)$ & $G_{3}\left(q^{2}\right)$ \\
\hline $\mathcal{F}\left(q^{2}=0\right)$ & $1.220 \pm 0.293$ & $-0.256 \pm 0.061$ & $-0.421 \pm 0.101$ & $0.751 \pm 0.180$ & $-0.156 \pm 0.037$ & $0.320 \pm 0.077$ \\
\hline$\xi_{1}$ & 1.03 & 2.17 & 2.18 & 1.41 & 1.46 & 2.36 \\
\hline$\xi_{2}$ & -4.60 & -8.63 & -1.02 & -3.30 & -6.50 & -2.90 \\
\hline$\xi_{3}$ & 28 & 51.40 & 18.12 & 21.90 & 41.20 & 28.20 \\
\hline$\xi_{4}$ & -53 & -85.2 & -32 & -40.10 & -74.82 & -45.2 \\
\hline
\end{tabular}

To find the lower bounds on the Borel parameters we demand that the perturbative part exceeds the total nonperturbative contributions and the highest order operator constitutes maximally $10 \%$ of the total contribution in each case. With these requirements, the working regions for the Borel parameters are found to be

$$
6 \mathrm{GeV}^{2} \leq M^{2} \leq 10 \mathrm{GeV}^{2},
$$

and

$$
4 \mathrm{GeV}^{2} \leq M^{\prime 2} \leq 6 \mathrm{GeV}^{2}
$$

The aforesaid intervals for the Borel and threshold parameters prompts the below window to the parameter $\beta$ :

$$
-0.5 \leq x \leq+0.5
$$

where we utilize $x=\cos \theta$ with $\theta=\tan ^{-1} \beta$ to examine the full region i.e., $-\infty$ to $\infty$ for $\beta$ by changing $x$ in the interval $[-1,1]$. Note that the Ioffe current with $x=-0.71$ stays out of the trustworthy region in this evaluation.

Having determined the working regions for the auxiliary parameters we proceed to find the behaviors of the form factors in terms of $q^{2}$. Our analysis shows that the form factors are well fitted to the function

$\mathcal{F}\left(q^{2}\right)=\frac{\mathcal{F}(0)}{\left(1-\xi_{1} \frac{q^{2}}{m_{\Lambda_{b}}^{2}}+\xi_{2} \frac{q^{4}}{m_{\Lambda_{b}}^{4}}+\xi_{3} \frac{q^{6}}{m_{\Lambda_{b}}^{6}}+\xi_{4} \frac{q^{8}}{m_{\Lambda_{b}}^{8}}\right)}$,
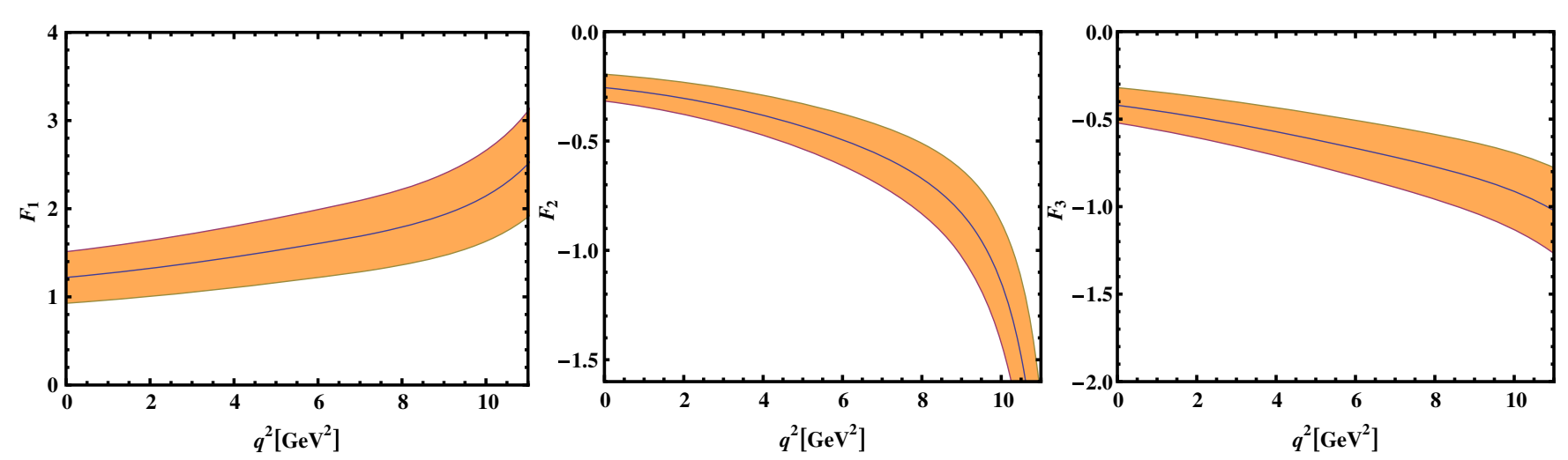

FIG. 1. $F_{1}, F_{2}$, and $F_{3}$ form factors as a function of $q^{2}$ at average values of auxiliary parameters.

where the average values of the parameters, $\mathcal{F}(0), \xi_{1}, \xi_{2}$, $\xi_{3}$, and $\xi_{4}$ for $\Lambda_{b} \rightarrow \Lambda_{c} \ell \bar{\nu}$ transition are presented in the Table II. Note that to find the average values for different parameters presented in Table II, we first find their values at different values of the auxiliary parameters $M^{2}, M^{\prime 2}, s_{0}, s_{0}^{\prime}$ and $x$ in their working intervals then take the average of the obtained values at various points.

Figures 1 and 2 show the dependence of the form factors $F_{i}$ and $G_{i}$ on $q^{2}$ in its allowed region, $m_{l}^{2} \leq q^{2} \leq\left(m_{\Lambda_{b}}-m_{\Lambda_{c}}\right)^{2}$ and at average values of auxiliary parameters. As is seen we encounter the uncertainties of the form factors in these figures. The solid lines show the average behavior of the form factors. From these figures we see that the form factors demonstrate a good behavior and gradually increase with increasing the transferred momentum squared. The fit functions of form factors will be used as the main input parameters to evaluate different physical observables in the next section.

\section{DECAY WIDTH AND BRANCHING RATIO OF $\Lambda_{b} \rightarrow \Lambda_{c} \ell \overline{\nu_{\ell}}$}

In this section we would like to evaluate the decay widths and branching fractions of the semileptonic $\Lambda_{b} \rightarrow \Lambda_{b} \ell \bar{\nu}_{\ell}$ transitions in all lepton channels. To this end we use the previously defined amplitude [see Eqs. (2) and (3)], i.e.,

$$
\mathcal{M}=\frac{G_{F}}{\sqrt{2}} V_{c b} \bar{l} \gamma^{\mu}\left(1-\gamma_{5}\right) \nu\left\langle\Lambda_{c}\left(p^{\prime}\right)\left|\bar{c} \gamma_{\mu}\left(1-\gamma_{5}\right) b\right| \Lambda_{b}(p)\right\rangle,
$$



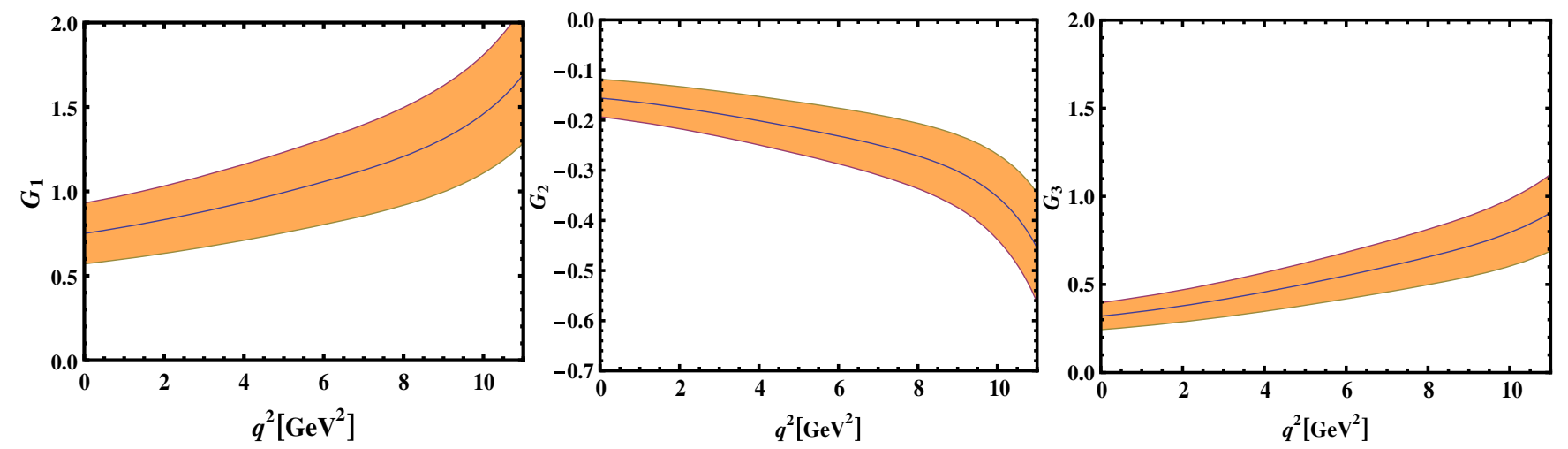

FIG. 2. $G_{1}, G_{2}$, and $G_{3}$ form factors as a function of $q^{2}$ at average values of auxiliary parameters.

as well as definitions of the transition matrix elements in terms of form factors from Eq. (4). By applying the Fermi golden rule and using the square of the above amplitude, after lengthy calculations according to the standard prescriptions, the angular distribution of the decay $\Lambda_{b} \rightarrow$ $\Lambda_{c} W^{-}\left(\rightarrow \ell^{-} \bar{\nu}_{\ell}\right)$ is obtained as (see also $[18,19,21,34]$ ):

$\frac{d \Gamma\left(\Lambda_{b} \rightarrow \Lambda_{c} \ell \bar{\nu}_{\ell}\right)}{d q^{2} d \cos \theta}=\frac{G_{F}^{2}}{(2 \pi)^{3}}\left|V_{c b}\right|^{2} \frac{\lambda^{1 / 2}\left(q^{2}-m_{\ell}^{2}\right)^{2}}{48 m_{\Lambda_{b}}^{3} q^{2}} W\left(\theta, q^{2}\right)$,

where, $m_{\ell}$ is the lepton mass, $\theta$ is the angle between the momenta of lepton $\ell^{-}$and $W^{-}$,

$$
\begin{aligned}
\lambda & \equiv \lambda\left(m_{\Lambda_{b}}^{2}, m_{\Lambda_{c}}^{2}, q^{2}\right) \\
& =m_{\Lambda_{b}}^{4}+m_{\Lambda_{c}}^{4}+q^{4}-2\left(m_{\Lambda_{b}}^{2} m_{\Lambda_{c}}^{2}+m_{\Lambda_{c}}^{2} q^{2}+m_{\Lambda_{b}}^{2} q^{2}\right),
\end{aligned}
$$

and

$$
\begin{aligned}
& W\left(\theta, q^{2}\right)=\frac{3}{8}\left\{\left(1+\cos ^{2} \theta\right) \mathbb{\sharp}_{U}\left(q^{2}\right)-2 \cos \theta \mathbb{H}_{P}\left(q^{2}\right)\right. \\
& +2 \sin ^{2} \theta \mathbb{H}_{L}\left(q^{2}\right)+\frac{m_{\ell}^{2}}{q^{2}}\left(2 \mathbb{H}_{S}\left(q^{2}\right)\right. \\
& +\sin ^{2} \theta \mathbb{}_{U}\left(q^{2}\right)+2 \cos ^{2} \theta \mathbb{Z}_{L}\left(q^{2}\right) \\
& \left.\left.-4 \cos \theta \mathbb{I}_{S L}\left(q^{2}\right)\right)\right\} \text {. }
\end{aligned}
$$

The parity conserving helicity structures entering the above equations are defined [18] as

$$
\begin{aligned}
\mathbb{M}_{U}\left(q^{2}\right) & =\left|\mathcal{H}_{+1 / 2,+1}\right|^{2}+\left|\mathcal{H}_{-1 / 2,-1}\right|^{2}, \\
\mathbb{M}_{L}\left(q^{2}\right) & =\left|\mathcal{H}_{+1 / 2,0}\right|^{2}+\left|\mathcal{H}_{-1 / 2,0}\right|^{2}, \\
\mathbb{U}_{S}\left(q^{2}\right) & =\left|\mathcal{H}_{+1 / 2, t}\right|^{2}+\left|\mathcal{H}_{-1 / 2, t}\right|^{2}, \\
\mathbb{U}_{S L}\left(q^{2}\right) & =\operatorname{Re}\left(\mathcal{H}_{+1 / 2,0} \mathcal{H}_{+1 / 2, t}^{\dagger}+\mathcal{H}_{-1 / 2,0} \mathcal{H}_{-1 / 2, t}^{\dagger}\right),
\end{aligned}
$$

where, the parity violating helicity structure is given by [18]

$$
\mathbb{M}_{P}\left(q^{2}\right)=\left|\mathcal{H}_{+1 / 2,+1}\right|^{2}-\left|\mathcal{H}_{-1 / 2,-1}\right|^{2}
$$

The helicity amplitudes entered the above relations are also defined in terms of the corresponding form factors as $[18,34]$

$$
\begin{aligned}
\mathcal{H}_{+1 / 2,0}^{V, A}= & \frac{1}{\sqrt{q^{2}}} \sqrt{2 m_{\Lambda_{b}} m_{\Lambda_{c}}(w \mp 1)} \\
& \times\left[\left(m_{\Lambda_{b}} \pm m_{\Lambda_{c}}\right) F_{1}^{V, A}(w) \pm m_{\Lambda_{c}}(w \pm 1) F_{2}^{V, A}(w)\right. \\
& \left. \pm m_{\Lambda_{b}}(w \pm 1) F_{3}^{V, A}(w)\right], \\
\mathcal{H}_{+1 / 2,1}^{V, A}= & -2 \sqrt{m_{\Lambda_{b}} m_{\Lambda_{c}}(w \mp 1)} F_{1}^{V, A}(w), \\
\mathcal{H}_{+1 / 2, t}^{V, A}= & \frac{1}{\sqrt{q^{2}}} \sqrt{2 m_{\Lambda_{b}} m_{\Lambda_{c}}(w \pm 1)} \\
& \times\left[\left(m_{\Lambda_{b}} \mp m_{\Lambda_{c}}\right) F_{1}^{V, A}(w) \pm\left(m_{\Lambda_{b}}-m_{\Lambda_{c}} w\right) F_{2}^{V, A}(w)\right. \\
& \left. \pm\left(m_{\Lambda_{b}} w-m_{\Lambda_{c}}\right) F_{3}^{V, A}(w)\right],
\end{aligned}
$$

with

$$
w=\frac{m_{\Lambda_{b}}^{2}+m_{\Lambda_{c}}^{2}-q^{2}}{2 m_{\Lambda_{b}} m_{\Lambda_{c}}},
$$

where the upper and lower sings corresponds to $V$ and $A$, respectively and $F_{i}^{V} \equiv F_{i}, F_{i}^{A} \equiv G_{i}(i=1,2,3)$. Here, $H_{\lambda^{\prime}, \lambda_{W}}^{V, A}$ are the helicity amplitudes for weak transitions generated by vector and axial vector currents, while $\lambda^{\prime}$ and $\lambda_{W}$ are the helicities of the final baryon and the virtual $W$-boson, respectively. $\lambda_{W}=t$ for total angular momentum $J=0$, with $\mathrm{t}$ meaning temporal. The amplitudes for negative values of the helicities can be purchased using [18]

$$
H_{-\lambda^{\prime},-\lambda_{W}}^{V, A}= \pm H_{\lambda^{\prime}, \lambda_{W}}^{V, A}
$$


TABLE III. Decay width (in GeV) and branching ratio of the semileptonic $\Lambda_{b} \rightarrow \Lambda_{c} \ell \bar{\nu}_{\ell}$ transition.

\begin{tabular}{|c|c|c|c|c|c|c|c|c|}
\hline Parameter & Present Work & {$[18]$} & {$[19,21]$} & {$[22]$} & {$[20]$} & {$[23]$} & {$[16]$} & Experiment [29] \\
\hline \multicolumn{9}{|c|}{$\overline{\Lambda_{b} \rightarrow \Lambda_{c}(e, \mu) \bar{\nu}_{(e, \mu)}}$} \\
\hline$\Gamma \times 10^{14}$ & $2.32 \pm 0.64$ & 2.91 & & 3.03 & & & 2.23 & \\
\hline $\operatorname{Br}(\%)$ & $6.04 \pm 1.70$ & 6.48 & 6.9 & & 4.83 & 6.3 & & $6.2_{-1.3}^{+1.4}$ \\
\hline \multicolumn{9}{|l|}{$\Lambda_{b} \rightarrow \Lambda_{c} \tau \bar{\nu}_{\tau}$} \\
\hline $\mathrm{Br}(\%)$ & $1.87 \pm 0.52$ & 2.03 & 2.0 & & 1.63 & & & \\
\hline
\end{tabular}

and the total helicity amplitude for the vector-axial vector current can be written as [18]

$$
\mathcal{H}_{\lambda^{\prime}, \lambda_{W}}=\mathcal{H}_{\lambda^{\prime}, \lambda_{W}}^{V}-\mathcal{H}_{\lambda^{\prime}, \lambda_{W}}^{A}
$$

Performing the integral over $\cos \theta$ in Eq. (22), the differential decay width is obtained as [18,21,34]:

$$
\frac{d \Gamma\left(\Lambda_{b} \rightarrow \Lambda_{c} \ell \bar{\nu}_{\ell}\right)}{d q^{2}}=\frac{G_{F}^{2}}{(2 \pi)^{3}}\left|V_{c b}\right|^{2} \frac{\lambda^{1 / 2}\left(q^{2}-m_{\ell}^{2}\right)^{2}}{48 m_{\Lambda_{b}}^{3} q^{2}} \mathbb{H}_{\mathrm{tot}}\left(q^{2}\right)
$$

where

$$
\mathbb{H}_{\mathrm{tot}}\left(q^{2}\right)=\left[\mathbb{H}_{U}\left(q^{2}\right)+\mathbb{H}_{L}\left(q^{2}\right)\right]\left(1+\frac{m_{\ell}^{2}}{2 q^{2}}\right)+\frac{3 m_{\ell}^{2}}{2 q^{2}} \mathbb{H}_{S}\left(q^{2}\right) .
$$

Now, using the fit functions of the form factors previously found and other inputs we are able to estimate the decay width and branching ratios of the transitions under consideration. The numerical values for the decay widths and branching ratios at different channels are shown in Table III. In this table, we also present the predictions of other theoretical methods (in some cases we have changed the original unit to $\mathrm{GeV}$ ) as well as the existing experimental data. From this table we see that the order of magnitude for the widths and branching fractions from different theoretical predictions are the same, though they show considerable differences in values in some cases. Our result on the branching ratio in $e, \mu$ channel is in nice agreement with average experimental value presented in PDG [29]. Our predictions at $\tau$ channel can be checked by future experiments.

As a final task we would like to report the ratio of branching fraction in $\tau$ channel to that of the $e, \mu$ :

$$
R=\frac{\operatorname{Br}\left[\Lambda_{b} \rightarrow \Lambda_{c}(e, \mu) \bar{\nu}_{(e, \mu)}\right]}{\operatorname{Br}\left[\Lambda_{b} \rightarrow \Lambda_{c} \tau \bar{\nu}_{\tau}\right]}=0.31 \pm 0.11,
$$

which may also be checked by future experiments.

\section{CONCLUSION}

The recent serious deviations of the experimental data from the theoretical productions made in the context of SM on the ratios of the branching fractions of the mesonic $B \rightarrow D^{(*)}$ decays in $\tau$ channel to that of the $(e, \mu)$ have put this subject in the focus of the much attention. While direct searches end up with null results in the search of NP effects at different colliders, these can be considered as significant indications for the NP effects beyond the SM. The corresponding $b \rightarrow c \ell \bar{\nu}_{\ell}$ based transition at baryon sector that is possible to study in future experiments is the semileptonic $\Lambda_{b} \rightarrow \Lambda_{c} \ell \bar{\nu}_{\ell}$ transition. we shall look at different experiments whether similar deviations is the case in this transition or not? In this connection we studied this transition at all lepton channels by calculating the responsible form factors in full QCD. we used the fit functions of the form factors to estimate the corresponding decay rates and branching fractions. We found the ratio $R=\frac{\operatorname{Br}\left[\Lambda_{b} \rightarrow \Lambda_{c} \tau \bar{\nu}_{\tau}\right]}{\operatorname{Br}\left[\Lambda_{b} \rightarrow \Lambda_{c}(e, \mu) \bar{\nu}_{(e, \mu)}\right]}=0.31 \pm 0.11$, which may be checked in future experiments. If we observe serious deviations of data on this ratio from the SM predictions, like those of the mesonic channels, this will increase our desire to indirectly search for new physics effects in heavy hadronic decay channels.

\section{ACKNOWLEDGMENTS}

We would like to thank H. Sundu for useful discussions. Work of K. A. was partly financed by Doguş University through the Project No. BAP 2015-16-D1-B04.

\section{APPENDIX: THE SPECTRAL DENSITIES}

In the following we present the explicit forms of spectral densities corresponding to the form factor $F_{1}$ : 


$$
\begin{aligned}
\rho_{\nu^{\prime} \gamma_{\mu}}^{\text {Pert }}\left(s, s^{\prime}, q^{2}\right)= & \int_{0}^{1} d u \int_{0}^{1-u} d v\left[\beta ^ { 2 } \left\{\frac { 1 } { 1 5 3 6 \pi ^ { 4 } ( u + v - 1 ) ^ { 2 } } \left[-16 m_{b}^{3}(u-1) v(u+v)+16 m_{b}^{2} m_{c} u v(u+v)\right.\right.\right. \\
& +m_{b}\left(-7 s^{\prime}(u-1)^{2} u^{2}+(u-1) u\left[8\left(s+s^{\prime}\right)-7\left(s+2 s^{\prime}\right) u+q^{2}(23 u-8)\right] v\right. \\
& \left.+\left[-9 s+8\left(-3 q^{2}+3 s+s^{\prime}\right) u+\left(23 q^{2}-7\left(2 s+s^{\prime}\right)\right) u^{2}\right] v^{2}+s(9-7 u) v^{3}-16 m_{c}^{2}(u-1) u(u+v)\right) \\
& +m_{c} u\left(s(u+v-1)[u+7 u v+v(2+7 v)]+u\left[16 m_{c}^{2}(u+v)+s^{\prime}(u+v-1)(1+7 u+7 v)\right]\right. \\
& \left.\left.\left.-q^{2} u[u+23 u v+v(23 v-6)-1]\right)\right]\right\} \\
& +\frac{1}{768 \pi^{4}(u+v-1)^{2}}\left\{-12 m_{b}^{3}(u-1) v(u+v)+12 m_{b}^{2} m_{c} u v(u+v)+m_{b}\left[-7 s^{\prime}(u-1)^{2} u^{2}\right.\right. \\
& +(u-1) u\left(6\left(s+s^{\prime}\right)-7\left(s+2 s^{\prime}\right) u+q^{2}\left(19 u_{6}\right)\right) v+\left(-5 s+6\left(-3 q^{2}+3 s+s^{\prime}\right) u\right. \\
& \left.\left.+\left[19 q^{2}-7\left(2 s+s^{\prime}\right)\right] u^{2}\right) v^{2}+s(5-7 u) v^{3}-12 m_{c}^{2}(u-1) u(u+v)\right] \\
& +m_{c} u\left[q^{2} u(u-19 u v+(8-19 v) v-1)+s(u+v-1)(v(7 v-2)+u(7 v-1))\right. \\
& \left.\left.\left.+u\left(12 m_{c}^{2}(u+v)+s^{\prime}(u+v-1)(7 u+7 v-1)\right)\right]\right\}\right] \Theta\left[L\left(s, s^{\prime}, q^{2}\right)\right], \\
\rho_{p^{\prime} \gamma_{\mu}}^{3}\left(s, s^{\prime}, q^{2}\right)= & \frac{1}{192 \pi^{2}} \int_{0}^{1} d u \int_{0}^{1-u} d v\left\{\langle d \bar{d}\rangle\left(2 \beta^{2}(4+3 u)-\beta(4-12 u) 12 u\right)+\langle u \bar{u}\rangle\left(-3 \beta^{2}(2+u)+4 \beta(3 u-1)+2\right)\right\} \\
& \times \Theta\left[L\left(s, s^{\prime}, q^{2}\right)\right], \quad \rho_{p^{\prime} \gamma_{\mu}}\left(s, s^{\prime}, q^{2}\right)=0, \\
& \rho_{p^{\prime} \gamma_{\mu}}^{5}\left(s, s^{\prime}, q^{2}\right)=0,
\end{aligned}
$$

where,

$$
L\left(s, s^{\prime}, q^{2}\right)=-m_{c}^{2} u+s^{\prime} u-s^{\prime} u^{2}-m_{b}^{2} v+s v+q^{2} u v-s u v-s^{\prime} u v-s v^{2}
$$

with $\Theta[. .$.$] being the unit-step function.$

[1] J. P. Lees et al. (BABAR Collaboration), Evidence for an Excess of $\bar{B} \rightarrow D^{(*)} \tau^{-} \bar{\nu}_{\tau}$ Decays, Phys. Rev. Lett. 109, 101802 (2012).

[2] S. Fajfer, J. F. Kamenik, and I. Nisandzic, On the $B \rightarrow D^{*} \tau \bar{\nu}_{\tau}$ sensitivity to new physics, Phys. Rev. D 85, 094025 (2012).

[3] S. Fajfer, J. F. Kamenik, I. Nisandzic, and J. Zupan, Implications of Lepton Flavor Universality Violations in B Decays, Phys. Rev. Lett. 109, 161801 (2012).

[4] R. Aaij et al. (LHCb Collaboration), Test of Lepton Universality Using $B^{+} \rightarrow K^{+} \ell^{+} \ell^{-}$Decays, Phys. Rev. Lett. 113, 151601 (2014).

[5] R. Aaij et al. (LHCb Collaboration), Test of lepton universality with $B^{0} \rightarrow K^{* 0} \ell^{+} \ell^{-}$decays, J. High Energy Phys. 08 (2017) 055.
[6] K. Azizi, A. T. Olgun, and Z. Tavukoglu, Impact of scalar leptoquarks on heavy baryonic decays, Adv. High Energy Phys. 2017, 7435876 (2017).

[7] T. M. Aliev, K. Azizi, and M. Savci, Analysis of the $\Lambda_{b} \rightarrow$ $\Lambda \ell^{+} \ell^{-}$decay in QCD, Phys. Rev. D 81, 056006 (2010).

[8] T. Aaltonen et al. (CDF Collaboration), Observation of the Baryonic Flavor-Changing Neutral Current Decay $\Lambda_{b} \rightarrow \Lambda \mu^{+} \mu^{-}$, Phys. Rev. Lett. 107, 201802 (2011).

[9] R. Aaij et al. (LHCb Collaboration), Differential branching fraction and angular analysis of $\Lambda_{b}^{0} \rightarrow \Lambda \mu^{+} \mu^{-}$decays, J. High Energy Phys. 06 (2015) 115.

[10] W. Detmold and S. Meinel, $\Lambda_{b} \rightarrow \Lambda \ell^{+} \ell^{-}$form factors, differential branching fraction, and angular observables from lattice QCD with relativistic $b$ quarks, Phys. Rev. D 93, 074501 (2016). 
[11] R. Aaij et al. (LHCb Collaboration), Measurement of matter-antimatter differences in beauty baryon decays, Nat. Phys. 13, 391 (2017).

[12] T. Feldmann and M. W. Y. Yip, Form factors for $\Lambda_{b} \rightarrow \Lambda$ transitions in SCET, Phys. Rev. D 85, 014035 (2012); Erratum, Phys. Rev. D 86, 079901(E) (2012).

[13] R. S. Marques de Carvalho, F. S. Navarra, M. Nielsen, E. Ferreira, and H. G. Dosch, Form-factors and decay rates for heavy Lambda semileptonic decays from QCD sum rules, Phys. Rev. D 60, 034009 (1999).

[14] T. M. Aliev, A. Ozpineci, and M. Savci, Model independent analysis of $\Lambda$ baryon polarizations in $\Lambda_{b} \rightarrow \Lambda l^{+} l^{-}$decay, Phys. Rev. D 67, 035007 (2003).

[15] K. Azizi, M. Bayar, Y. Sarac, and H. Sundu, Semileptonic $\Lambda_{b, c}$ to nucleon transitions in full QCD at light cone, Phys. Rev. D 80, 096007 (2009).

[16] W. Detmold, C. Lehner, and S. Meinel, $\Lambda_{b} \rightarrow p \ell^{-} \bar{\nu}_{\ell}$ and $\Lambda_{b} \rightarrow \Lambda_{c} \ell^{-} \bar{\nu}_{\ell}$ form factors from lattice QCD with relativistic heavy quarks, Phys. Rev. D 92, 034503 (2015).

[17] T. Mannel and D. van Dyk, Zero-recoil sum rules for $\Lambda_{b} \rightarrow \Lambda_{c}$ form factors, Phys. Lett. B 751, 48 (2015).

[18] R. N. Faustov and V. O. Galkin, Semileptonic decays of $\Lambda_{b}$ baryons in the relativistic quark model, Phys. Rev. D 94, 073008 (2016).

[19] T. Gutsche, M. A. Ivanov, J. G. Körner, V. E. Lyubovitskij, P. Santorelli, and N. Habyl, Semileptonic decay $\Lambda_{b} \rightarrow$ $\Lambda_{c}+\tau^{-}+\overline{\nu_{\tau}}$ in the covariant confined quark model, Phys. Rev. D 91, 074001 (2015); Erratum, Phys. Rev. D 91, 119907(E) (2015).

[20] R. Dutta, $\Lambda_{b} \rightarrow\left(\Lambda_{c}, p\right) \tau \nu$ decays within standard model and beyond, Phys. Rev. D 93, 054003 (2016).

[21] T. Gutsche, M. A. Ivanov, J. G. Körner, V. E. Lyubovitskij, and P. Santorelli, Heavy-to-light semileptonic decays of $\Lambda_{b}$ and $\Lambda_{c}$ baryons in the covariant confined quark model, Phys. Rev. D 90, 114033 (2014); Semileptonic decays $\Lambda_{c}^{+} \rightarrow \Lambda \ell^{+} \nu_{\ell}(\ell=e, \mu)$ in the covariant quark model and comparison with the new absolute branching fraction measurements of Belle and BESIII, Phys. Rev. D, 93, 034008 (2016).
[22] M. Pervin, W. Roberts, and S. Capstick, Semileptonic decays of heavy lambda baryons in a quark model, Phys. Rev. C 72, 035201 (2005).

[23] H. W. Ke, X. Q. Li, and Z. T. Wei, Diquarks and $\Lambda_{b} \rightarrow \Lambda_{c}$ weak decays, Phys. Rev. D 77, 014020 (2008).

[24] A. Datta, S. Kamali, S. Meinel, and A. Rashed, Phenomenology of $\Lambda_{b} \rightarrow \Lambda_{c} \tau \bar{\nu}_{\tau}$ using lattice QCD calculations, J. High Energy Phys. 08 (2017) 131.

[25] Y. B. Dai, C. S. Huang, M. Q. Huang, and C. Liu, QCD sum rule analysis for the $\Lambda_{b} \rightarrow \Lambda_{c}$ semileptonic decay, Phys. Lett. B 387, 379 (1996).

[26] S. Shivashankara, W. Wu, and A. Datta, $\Lambda_{b} \rightarrow \Lambda_{c} \tau \bar{\nu}_{\tau}$ decay in the standard model and with new physics, Phys. Rev. D 91, 115003 (2015).

[27] M. A. Shifman, A. I. Vainshtein, and V. I. Zakharov, QCD and resonance physics: Applications, Nucl. Phys. B147, 448 (1979).

[28] L. J. Reinders, H. Rubinstein, and S. Yazaki, Hadron properties from QCD sum rules, Phys. Rep. 127, 1 (1985).

[29] C. Patrignani et al. (Particle Data Group), Review of particle physics, Chin. Phys. C 40, 100001 (2016) and 2017 update.

[30] H. G. Dosch, M. Jamin, and S. Narison, Baryon masses and flavor symmetry breaking of chiral condensates, Phys. Lett. B 220, 251 (1989).

[31] V. M. Belyaev and B. L. Ioffe, Zh. Eksp. Teor. Fiz. 84, 1236 (1983) [Determination of the baryon mass and baryon resonances from the quantum-chromodynamics sum rule. Strange baryons, Sov. Phys. JETP 57, 716 (1983)].

[32] B. L. Ioffe, QCD at low energies, Prog. Part. Nucl. Phys. 56, 232 (2006).

[33] E. M. Henley, W. Y. P. Hwang, and L. S. Kisslinger, Nonleptonic hyperon decays with QCD sum rules, Nucl. Phys. A706, 163 (2002).

[34] P. Bialas, J. G. Körner, M. Kramer, and K. Zalewski, Joint angular decay distributions in exclusive weak decays of heavy mesons and baryons, Z. Phys. C 57, 115 (1993). 